\section{The complete portfolio of implant solutions}

Delivering fantastic results on their own and even better results when used together, the complete portfolio of implant solutions from Nobel Biocare is a must-have for any clinician looking to take their implantology to the next level.

The cutting-edge range includes:


- NobelActive Wide Platform and NobelParallel Conical Connection implants - for outstanding primary stability and predictability of posterior implants

- Angulated Screw Channel (ASC) abutment and Omnigrip tooling - offering exciting new restorative possibilities in the anterior and posterior region

- Full Contour Zirconia (FCZ) Implant Crown - delivering exceptional aesthetics and strength

- All-On-4 treatment concept - the popular concept that enables restoration of complete arches in a single day

- Creos xenoprotect - the resorbable collagen membrane designed for bone regeneration and guided tissue regeneration procedures

- NobelClinician - the software every implant dentist needs for accurate treatment planning and surgery.

To find out more about how these solutions could enhance your implant dentistry, contact Nobel Biocare today on 02087563300 or visit www.nobelbiocare.com.

\section{Award-winning easy access tool}

The new Walser Dental Matrix for last teeth and extra large molars has been recognised with the 'Best of' Industry Award 2016.

The matrix for last teeth is applied with just one hand movement within seconds and fits automatically disto-cervically around the tooth.

Patients are no longer inclined to gag, and there is no longer the necessity for removing composite in places that are difficult to access.

\section{This matrix}

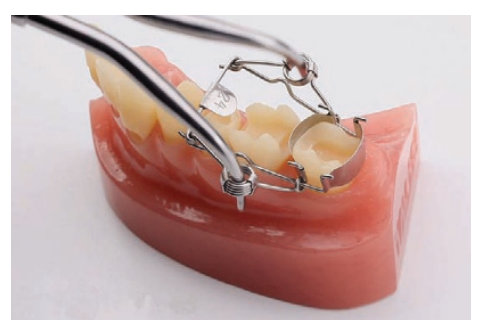

convinced the jury of

the Industry Awards in particular because of its value for money, it saves time, and it is based on the ecological advantages of Walser matrices: it can be used and sterilised many times and never loses its consistently high spring force.

www.walser-dental.com.

\section{Your total endo solution}

Simplify your endodontic shaping and reduce costs with the One Shape. Safe and effective, One Shape is the latest innovation from Micro-Mega: the company with over 100 years of endodontic experience.

One Shape features a variable asymmetrical cross section allowing for safe, effective debris removal and great flexibility. A variable pitch ensures reduced screwing effect which creates a smooth procedure for the clinician. The single file type means easy stock management at the surgery and with sterile blister packaging the instruments are ready to use directly from the pack. Procedure packs are also available which provide a complete set of instruments for a treatment.

For further information visit www.js-davis. co.uk or call 01438 747344 .

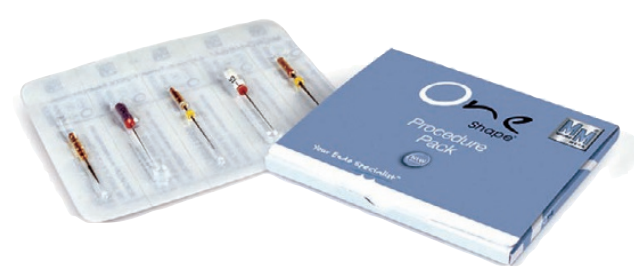

